\title{
Minimization of Viscous Fluid Fingering: A Variational Scheme for Optimal Flow Rates
}

\author{
Eduardo O. Dias, ${ }^{1}$ Enrique Alvarez-Lacalle, ${ }^{2}$ Márcio S. Carvalho, ${ }^{3}$ and José A. Miranda ${ }^{1, *}$ \\ ${ }^{1}$ Departamento de Física, Universidade Federal de Pernambuco, Recife, Pernambuco 50670-901, Brazil \\ ${ }^{2}$ Departament de Fisica Aplicada, Universitat Politecnica de Catalunya, Av. Doctor Marañón 44-50, Barcelona 08028, Spain \\ ${ }^{3}$ Departamento de Engenharia Mecânica, Pontifícia Universidade Católica do Rio de Janeiro,
}

Rio de Janeiro, Rio de Janeiro 22453-900, Brazil

(Received 5 June 2012; published 5 October 2012)

\begin{abstract}
Conventional viscous fingering flow in radial Hele-Shaw cells employs a constant injection rate, resulting in the emergence of branched interfacial shapes. The search for mechanisms to prevent the development of these bifurcated morphologies is relevant to a number of areas in science and technology. A challenging problem is how best to choose the pumping rate in order to restrain the growth of interfacial amplitudes. We use an analytical variational scheme to look for the precise functional form of such an optimal flow rate. We find it increases linearly with time in a specific manner so that interface disturbances are minimized. Experiments and nonlinear numerical simulations support the effectiveness of this particularly simple, but nontrivial, pattern controlling process.
\end{abstract}

DOI: 10.1103/PhysRevLett.109.144502

PACS numbers: 47.15.gp, 47.20.Ma, 47.55.N-

In their pioneering work [1], Saffman and Taylor observed that, when a fluid is displaced by another of lower viscosity in the confined geometry of a Hele-Shaw (HS) cell, the fluid-fluid interface may become unstable. This characterizes the viscous fingering (VF) instability which gives rise to complex interfacial morphologies. This fluid dynamic problem has been actively studied over half a century [2], and is an archetype for a wide range of fields, including research in oil recovery processes [3], fluid mixing [4], flow in granular media [5], microdischarges in plasmas [6], and biodynamics of cell fragmentation [7].

The radial flow setup of the Saffman-Taylor problem [8-11] has provided important insights into fundamental aspects of branching patterns. It takes place when the less viscous fluid is radially injected under constant injection rate, producing fingerlike structures which tend to split at their tips. Despite their visual appeal and physical relevance, the emergence of a ramified fingering configuration is not always desirable. For instance, it is well known that viscous fingering is a major factor in reducing oil recovery from underground petroleum reservoirs [3]. Therefore, it is of scientific and technological importance to investigate how to control, and eventually suppress, the growth of bifurcated patterns.

Controlling of radial VF has generated considerable interest in recent years [12-19]. These studies propose different strategies to avoid the appearance of branched morphologies. A first set of investigations [12-17] was not quite able to eliminate the fingers, but replaced typical convoluted forms by $n$-fold structures, where sizable fingers persist but do not bifurcate. This process conveniently determines the number of emerging fingers, keeping them fixed as the interface evolves. Regardless of whether the fluids are miscible or immiscible, and if the HS cell is flat [12-15], this can be accomplished by considering a time-dependent injection rate proportional to $t^{-1 / 3}$. Similar injection controlling protocols have been proved effective to dictate the final number of fingers even if the HS cell is nonflat [16], and also when a gel-like phase is formed at the interface due to chemical reactions between the fluids [17].

A second line of research $[18,19]$ searches for mechanisms that are capable not only of restraining densebranching formation in radial Hele-Shaw cells, but that also unfavors the establishment of interfacial deformations. In this context the ultimate goal would be to obtain front propagation in the form of nearly stable, axisymmetric interfaces. This has been achieved by utilizing a twostage piecewise constant injection process which applies a relatively low injection rate followed by a proper, stronger injection stage [18]. An alternative suppression method [19] employs a standard constant pumping rate, but substitutes the originally rigid HS upper plate with an elastic membrane. In this case, the onset of instability is deferred due to the membrane elastic distortions which reduce destabilizing pressure perturbations. Finally, a very recent work in rectangular Hele-Shaw cells addresses the possibility of controlling VF instabilities using flow geometry, through the introduction of a gradient in the flow passage [20].

Despite the ability of existing controlling methods $[18,19]$ to damp interfacial irregularities, a fundamental question still remains unanswered: if one wishes to inject a certain amount of fluid in a given time, what would be the optimal time-dependent injection rate $Q(t)$ for which the perturbation amplitudes could be truly minimized? Considering the multiplicity of possibilities for time-varying injection scenarios the response to this pertinent query is decidedly nontrivial.

In contrast to Refs. [12-19], we present a variational scheme which allows one to systematically search for the 
particular $Q(t)$ that leads to the prevention of the radial fingering instability. The stabilization strategy we propose does not require unconventional modifications of the traditional radial HS flow setup, and does not depend on the material properties of the fluids. It provides a simple and practical way, derived from first principles, to improve the efficiency and control of complex fingering phenomena. Our analytical results are supported by experiments and nonlinear numerical simulations.

Consider a radial HS cell comprising two close parallel glass plates of spacing $b$ containing two immiscible, incompressible, viscous fluids. The viscosities of the fluids are denoted as $\eta_{1}$ and $\eta_{2}$. Fluid 1 is injected into fluid 2 at a given injection rate $Q$ (area covered per unit time), which may depend on time. The fluids involved are Newtonian, and the surface tension between them is $\sigma$. Linear stability analysis of the problem [8] considers harmonic distortions of a nearly circular fluid-fluid interface whose radius evolves according to $\mathcal{R}(\theta, t)=R(t)+\zeta_{n}(t) \cos n \theta$, where the time-dependent unperturbed radius is $R(t)=\sqrt{R_{0}^{2}+(1 / \pi) \int_{0}^{t} Q\left(t^{\prime}\right) d t^{\prime}}, \theta$ represents the azimuthal angle, and $n$ are integer wave numbers. The unperturbed radius of the interface at $t=0$ is denoted by $R_{0}$. The Fourier perturbation amplitudes are given by [10]

$$
\zeta_{n}(t)=\zeta_{n}(0) \exp \{I(n, R, \dot{R})\}
$$

where

$$
I(n, R, \dot{R})=\int_{t_{c}(n)}^{t} \lambda(n, R, \dot{R}) d t^{\prime}
$$

with $t_{c}(n)$ being the time at which a mode $n$ becomes unstable $[\lambda(n)=0]$. It is assumed that $\zeta_{n}(t)=\zeta_{n}(0)$ if $0 \leq$ $t<t_{c}(n) . \dot{R}=Q(t) / 2 \pi R$ is the velocity of the unperturbed interface (the overdot denotes total time derivative), $\alpha=$ $b^{2} \sigma /\left[12\left(\eta_{1}+\eta_{2}\right)\right]$, and $\lambda(n, R, \dot{R})=(\dot{R} / R)(A|n|-1)-$ $\left(\alpha / R^{3}\right)|n|\left(n^{2}-1\right)$ is the linear growth rate, where $A=\left(\eta_{2}-\eta_{1}\right) /\left(\eta_{2}+\eta_{1}\right)$ is the viscosity contrast. For unstable situations $A>0$.

The mode of maximum growth rate can be obtained by setting $d \lambda(n, R, \dot{R}) / d n=0$, yielding

$$
n_{\max }(R, \dot{R})=\sqrt{\frac{1}{3}\left(1+\frac{A \dot{R} R^{2}}{\alpha}\right)} \approx \sqrt{\frac{A \dot{R} R^{2}}{3 \alpha}} .
$$

For situations of interest regarding interfacial pattern formation, the approximation used in Eq. (3) is justified by the fact that in typical experimental conditions $[8-13,19]$ the parameter $\alpha$ is usually very small $\left(10^{-12} \leq \alpha \leq\right.$ $10^{-7} \mathrm{~m}^{3} / \mathrm{s}$ ), so that $A \dot{R} R^{2} / \alpha \gg 1$ for unstable growth of the interface.

Our main task is to minimize the perturbations amplitudes (1). This can be accomplished by extremizing the integral (2). Since $n_{\max }$ is the fastest growing mode, we focus on minimizing the integral

$$
I\left(n_{\max }, R, \dot{R}\right)=\int_{0}^{t} \lambda(R, \dot{R}) d t^{\prime},
$$

where $t_{c}\left(n_{\max }\right)=0$ and $\lambda(R, \dot{R})=2 A^{3 / 2} \dot{R}^{3 / 2} /(3 \sqrt{3 \alpha})-$ $\dot{R} / R$ only depends on $R$ and $\dot{R}$. Recall that we want to inject a certain amount of the less viscous fluid by keeping fixed initial and final radii, during a time interval $\left[0, t_{f}\right]$. Within this context, $I$ in Eq. (4) is the action, while $\lambda$ defines the Lagrangian of the system. Therefore, we actually have a variational problem which can be solved by using the Euler-Lagrange equation

$$
\frac{d}{d t}\left(\frac{\partial \lambda}{\partial \dot{R}}\right)=\frac{\partial \lambda}{\partial R},
$$

with fixed endpoints $R(t=0)=R_{0}$ and $R\left(t=t_{f}\right)=R_{f}$. Substitution of the growth rate $\lambda=\lambda(R, \dot{R})$ into Eq. (5) leads to a surprisingly simple differential equation $\ddot{R}=0$, whose solution is

$$
R(t)=R_{0}+\frac{\left(R_{f}-R_{0}\right)}{t_{f}} t .
$$

It is worthwhile to note that, if the approximation $A \dot{R} R^{2} / \alpha \gg 1$ is not used, the Euler-Lagrange equation yields a complicated nonlinear differential equation given by $A^{2} R^{5} \ddot{R}+2 \alpha A R^{2} \dot{R}+4 \alpha^{2}=0$. It turns out that for typical values of $\alpha$ the solution of this equation is indistinguishable from the straight line solution (6).

Since $Q(t)=2 \pi \dot{R} R$, from Eq. (6) we obtain an optimal injection rate that varies linearly with time as

$$
Q(t)=2 \pi \frac{\left(R_{f}-R_{0}\right)}{t_{f}}\left[R_{0}+\frac{\left(R_{f}-R_{0}\right)}{t_{f}} t\right] .
$$

This is a result that could hardly be anticipated: out of a multitude of possible choices for the time-dependent function $Q(t)$, the one that minimizes the perturbation amplitudes is given by a linearly increasing function as prescribed by Eq. (7) (see Fig. 1). In contrast to the

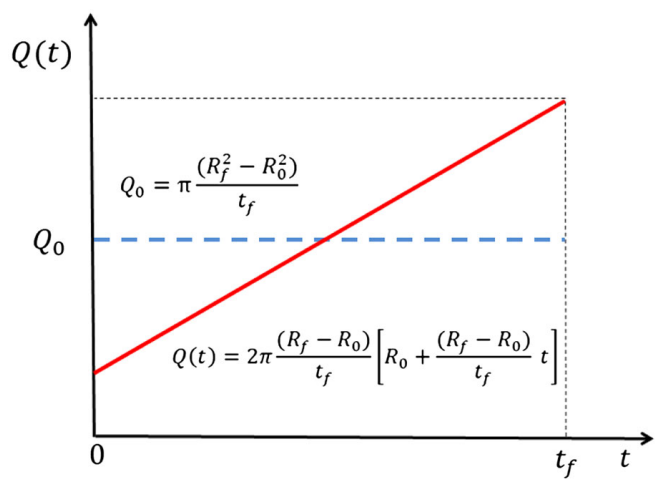

FIG. 1 (color online). Sketch of the injection rate as a function of time for the optimal injection $Q(t)$ (solid line). The equivalent constant injection rate $Q_{0}$ is represented by the horizontal dashed line. The total volume of injected fluid in the interval $\left[0, t_{f}\right]$ should be the same for both pumping rates. 
previous controlling strategies based on manipulations of the injection rate [12-17], it is interesting to note that our optimal pumping rate (7) does not depend on the material properties of the fluids. We emphasize that the key insight of our variational scheme is the analogy of the growth rate to a Lagrangian and the use of the corresponding EulerLagrange equation to find the optimal injection rate. This is fundamentally distinct from the so-called a variational "energy method" which is utilized to find stability regions in hydrodynamically unstable flows (Couette flow, Bénard flows, etc.) [21]. The energy method is much more involved than ours, and has a different physical motivation.

We have not included wetting effects in our study, but it is known that they can affect the VF instability [22]. A recent linear stability study [23] included wetting effects for radial flow in HS cells. From their results one can verify that wettability tends to decrease the value of the maximum growth rate, tending to stabilize the system. Besides, we have found that the fastest growing mode $\left(n_{\max }\right)$ does not vary much when wetting is added, implying that our Eq. (3) still holds. So, our variational scheme is certainly useful to search for a proper optimal flow rate when wetting is taken into account. However, we anticipate that a very complicated differential equation for the ideal $R(t)$ is obtained, so that analytical access to such ideal injection seems unlikely. We have also verified that, although not being the exact optimal injection when wetting is considered, our linearly increasing solution (7) leads to significant minimization of fingering when contrasted with the constant pumping procedure under the presence of wetting effects.

We proceed by examining both theoretically and experimentally the suitability of the interface stabilization process based on the optimal injection rate (7). For a given $R_{f}$ and $t_{f}$, traditional radial VF flow considers insertion of a specific volume of fluid at constant injection rate

$$
Q_{0}=\pi \frac{\left(R_{f}^{2}-R_{0}^{2}\right)}{t_{f}}
$$

We wish to compare the dynamic behavior and the resulting interface morphologies obtained by using the constant injection rate (8) and the ideal pumping rate (7) at $t=t_{f}$. A schematic representation of the behavior of $Q(t)$ and $Q_{0}$ as time progresses is given in Fig. 1. Notice from Eq. (8) that the relevant set of parameters to be fixed in the controlling mechanism could be either $R_{f}$ and $t_{f}$, or $Q_{0}$ and $t_{f}$.

The linear stability results are obtained by utilizing parameter values that are consistent with those used in typical experimental realizations of radial $\mathrm{HS}$ flows [8,9,12,13]: $R_{0}=4.5 \mathrm{~mm}, \quad b=1.0 \mathrm{~mm}, \quad \sigma=$ $25.0 \mathrm{mN} / \mathrm{m}, \eta_{2}=0.485 \mathrm{~Pa}$ s, with $\eta_{2} \gg \eta_{1}$. In addition, we set $R_{f}=4.8 \mathrm{~cm}$, and $t_{f}=18 \mathrm{~s}$. For the remainder of this work we focus on the most unstable situation where $A=1$, which is the most challenging to control. In Fig. 2 we plot the integrals given by Eq. (2) at $t=t_{f}$, for the optimal injection rate $I$ (solid curve), and for the equivalent

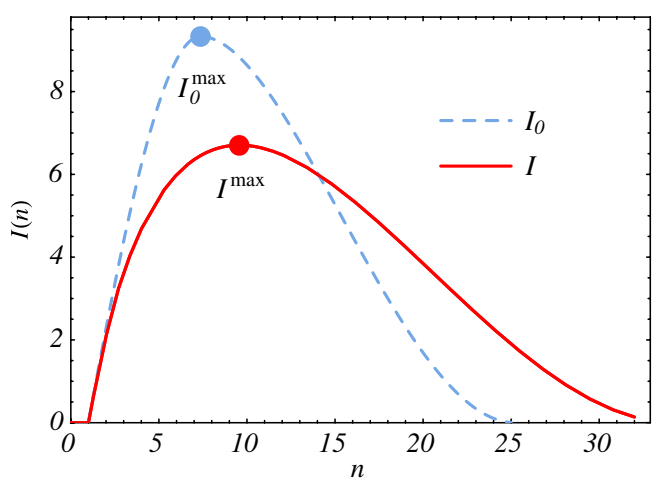

FIG. 2 (color online). The solid curve represents the integral $I$ as a function of mode $n$, calculated for the optimal injection rate (7). The dashed curve shows the corresponding quantity $I_{0}$ for the constant injection process (8).

constant pumping situation $I_{0}$ (dashed curve) as functions of the wave number $n$. By inspecting Fig. 2 we observe a significant reduction in the maximum value of $I(n)$ (denoted by $I^{\max }$ ) for the ideal injection rate case. Since the perturbation amplitudes $\zeta_{n}$ are related to the exponential of the integrals $I$ and $I_{0}$ [Eq. (1)], this implies that the decrease in the relative size of the largest fingers is of one order of magnitude. The physical explanation for the success of the ideal stabilization protocol [Eq. (7)] rests on the fact that initially the injection rate is sufficiently small (Fig. 1) so that propagation of a sizable unperturbed front is possible. As time progresses the injection rate increases considerably, but since it occurs at a large interfacial radius, injection is no longer able to promote a significant destabilization of the propagating boundary. This means that the onset of instability is delayed, and when it eventually takes place disturbances arise with a reduced growth rate.

It is important to address the generality of our linear stability results. In particular, it would be of interest to investigate the robustness of our optimal injection strategy when $Q_{0}$ or $t_{f}$ are increased. In fact, there is no upper bound value on $Q_{0}$ or $t_{f}$ for the minimization of finger lengths: under the ideal injection process the length of the fingers will always be significantly smaller than those produced under constant pumping. Regarding this point, we have verified that the difference between the maximum values of the integrals $I_{0}^{\max }-I^{\max }$ (see Fig. 2) grows as the injection rate $Q_{0}$ or the final time $t_{f}$ are increased. This issue can be quantitatively expressed in terms of a dimensionless final time, and a suitably defined modified capillary number for the system

$$
T_{f}=\frac{Q_{0} t_{f}}{\pi R_{0}^{2}}, \quad \text { and } \quad \mathrm{Ca}=\frac{12 \eta_{2} Q_{0}}{\pi R_{0} \sigma}\left(\frac{R_{0}}{b}\right)^{2},
$$

where lengths and velocities are rescaled by $R_{0}$, and $Q_{0} /\left(\pi R_{0}\right)$, respectively. Figure 3 depicts the ratio of the maximum perturbation amplitudes at $t=t_{f}, \zeta_{0} / \zeta \equiv$ $\exp \left[I_{0}^{\max }-I^{\max }\right]$ as a function of $T_{f}$ for two values of the 


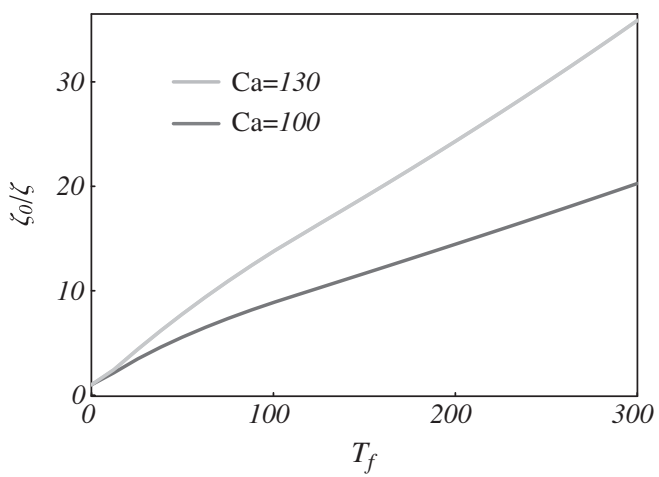

FIG. 3. Amplitude ratio $\zeta_{0} / \zeta$ as a function of $T_{f}$ for $\mathrm{Ca}=100$, 130. Here $\zeta_{0}(\zeta)$ denotes the maximum amplitude for constant (optimal) injection at $t=t_{f}$.

capillary number $\mathrm{Ca}$. From this figure it is clear that the ratio $\zeta_{0} / \zeta$ grows with increasing $T_{f}$ and $\mathrm{Ca}$. Of course, for large $T_{f}$ and $\mathrm{Ca}$, the final optimal injection interface can indeed present some undulations. However, the amplitudes of such perturbations are guaranteed to be considerably smaller than the ones obtained by the equivalent constant pumping process. Note that the condition $\dot{R} R^{2} / \alpha \gg 1$ mentioned at the beginning of the paper, can now be rewritten as $\mathrm{Ca} \dot{R} R^{2} \gg 1$. These linear stability predictions are consistent with our nonlinear simulations.

To validate the linear stability predictions, and also to access the system's response at more advanced time stages of the dynamics, we performed a series of experiments and simulations. The experimental apparatus consists of a radial HS cell made of two plexiglass plates $4.0 \mathrm{~cm}$ thick and $40.0 \mathrm{~cm}$ in diameter, uniformly separated by a narrow gap
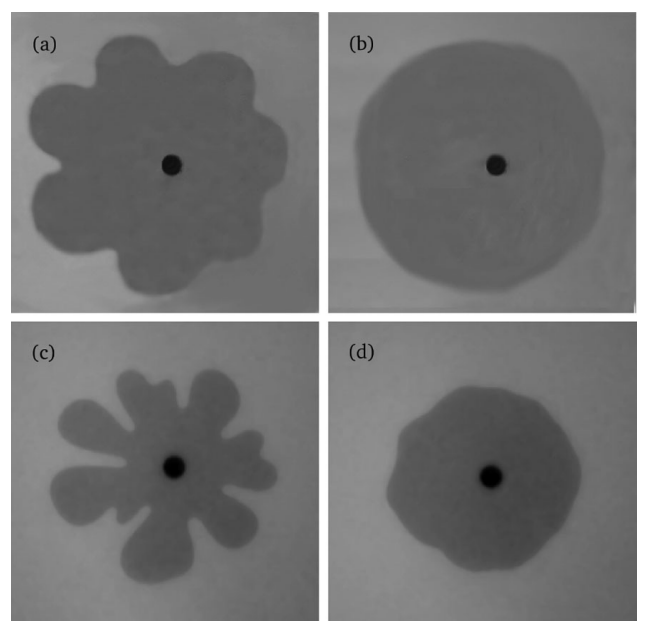

FIG. 4. Typical experimental patterns at time $t_{f}=20 \mathrm{~s}$ for (a) $Q_{0}=2.08 \mathrm{~cm}^{2} / \mathrm{s}$, and (b) its corresponding ideal injection rate. For (a) and (b) $T_{f}=65$ and $\mathrm{Ca}=112$. The experimental shapes shown in (c) and (d) are taken at $t_{f}=6.7 \mathrm{~s}$ for $Q_{0}=$ $3.75 \mathrm{~cm}^{2} / \mathrm{s}$, and for the equivalent optimal pumping rate, respectively. In (c) and (d) $T_{f}=40$ and $\mathrm{Ca}=200$. of thickness $b=2.0 \mathrm{~mm}$. A low viscosity fluid (water) is injected through a hole drilled at the center of the bottom plate, and a mineral oil (Talpa 30/Shell, $\eta_{2}=0.45 \mathrm{~Pa} \mathrm{~s}$ ) is used as the high viscosity fluid. The surface tension between the fluids is of the order of $10.0 \mathrm{mN} / \mathrm{m}$. A precision computer-controlled injection pump (Teledyne ISCO, model 500D) allowed both constant and time-dependent injection rates. Fingering events were video recorded using a charge coupled device video camera.

Representative experimental morphologies are illustrated in Fig. 4. It can be clearly seen that there exist two distinct classes of patterns. At constant injection rates [(a) and (c)] typical VF structures presenting decent-sized interfacial deformations are found. Considering the bluntness of the fingers, we can tell that in (a) we are at the beginning of the nonlinear stage. On the other hand, the larger length of the fingers, and the existence of finger tipsplitting indicate that in (c) we are at the fully nonlinear regime. However, if the ideal injection rates are employed [(b) and (d)] considerably less deformed patterns arise. Fingers are suppressed in (b), and their growth is strongly restrained in (d).

Finally, Fig. 5 presents boundary integral numerical simulations for the nonlinear evolution of the interface considering constant injection (left panel), and ideal injection (right panel). The same initial conditions have been used in both runs: a circle modulated by modes 2 to 10 , each one with an amplitude of $0.008 R_{0}$, and random phases. The numerical algorithm is described in detail in Ref. [24]. Figure 5 shows characteristic fanlike patterns with fingers of large lengths when injection is constant, and nearly circular shapes when the ideal controlling procedure is employed. The simulated morphologies resemble those obtained experimentally in Fig. 4.

These experimental and numerical findings substantiate our linear stability predictions, supporting the pertinence and usefulness of the proposed VF controlling process at nonlinear stages. All this provides a step forward from the current research on controllability toward ultimate control of complex VF patterns.
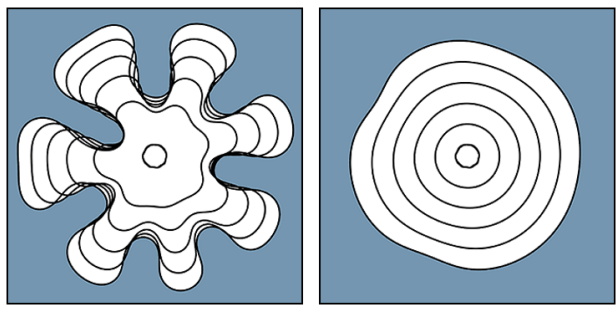

FIG. 5 (color online). Nonlinear numerical simulations depicting the time evolution of the interfacial patterns for constant injection $Q_{0}=1.25 \mathrm{~cm}^{2} / \mathrm{s}$ (left), and equivalent optimal injection (right). Here $t_{f}=240 \mathrm{~s}, R_{f}=9.8 \mathrm{~cm}$, and $R_{0}=1.0 \mathrm{~cm}$. The interfaces are plotted in intervals of $t_{f} / 5$. Here $T_{f}=95$ and $\mathrm{Ca}=95$. 
J. A. M., E. O. D., and M. S. C. thank CNPq for financial support. E. A. L. acknowledges support from Secretaría de Estado de IDI under project FIS2011-28820-C02-01. We are grateful to Michael Widom and Leonardo Cabral for helpful suggestions.

*jme@df.ufpe.br

[1] P. G. Saffman and G. I. Taylor, Proc. R. Soc. A 245, 312 (1958).

[2] K. V. McCloud and J. V. Maher, Phys. Rep. 260, 139 (1995); J. Casademunt, Chaos 14, 809 (2004).

[3] S. B. Gorell and G. M. Homsy, SIAM J. Appl. Math. 43, 79 (1983); J. P. Stokes, D. A. Weitz, J. P. Gollub, A. Dougherty, M. O. Robbins, P. M. Chaikin, and H.M. Lindsay, Phys. Rev. Lett. 57, 1718 (1986).

[4] B. Jha, L. Cueto-Felgueroso, and R. Juanes, Phys. Rev. Lett. 106, 194502 (2011).

[5] X. Cheng, L. Lu, A. Patterson, H. M. Jaeger, and S. R. Nagle, Nature Phys. 4, 234 (2008).

[6] H.-Y. Chu and H.-K. Lee, Phys. Rev. Lett. 107, 225001 (2011).

[7] A. C. Callan-Jones, J.-F. Joanny, and J. Prost, Phys. Rev. Lett. 100, 258106 (2008).

[8] L. Paterson, J. Fluid Mech. 113, 513 (1981).

[9] J. D. Chen, J. Fluid Mech. 201, 223 (1989).

[10] J.A. Miranda and M. Widom, Physica (Amsterdam) 120D, 315 (1998).
[11] J. Mathiesen, I. Procaccia, H.L. Swinney, and M. Thrasher, Europhys. Lett. 76, 257 (2006).

[12] S. W. Li, J. S. Lowengrub, J. Fontana, and P. PalffyMuhoray, Phys. Rev. Lett. 102, 174501 (2009).

[13] A. Leshchiner, M. Thrasher, M. B. Mineev-Weinstein, and H. L. Swinney, Phys. Rev. E 81, 016206 (2010).

[14] E. O. Dias and J. A. Miranda, Phys. Rev. E 81, 016312 (2010).

[15] C.-Y. Chen, C.-W. Huang, L.-C. Wang, and J. A. Miranda, Phys. Rev. E 82, 056308 (2010).

[16] L. dos Reis and J. A. Miranda, Phys. Rev. E 84, 066313 (2011).

[17] A. He, J. S. Lowengrub, and A. Belmonte, SIAM J. Appl. Math. 72, 842 (2012).

[18] E. O. Dias, F. Parisio, and J. A. Miranda, Phys. Rev. E 82, 067301 (2010).

[19] D. Pihler-Puzović, P. Illien, M. Heil, and A. Juel, Phys. Rev. Lett. 108, 074502 (2012).

[20] T. T. Al-Housseiny, P. A. Tsai, and H. A. Stone, Nature Phys. (in press).

[21] J. Serrin, Arch. Ration. Mech. Anal. 3, 1 (1959); D. D. Joseph, Arch. Ration. Mech. Anal. 22, 163 (1966); B. Straughan, The Energy Method, Stability, and Nonlinear Convection (Springer Verlag, New York, 1992).

[22] C. W. Park and G. M. Homsy, J. Fluid Mech. 139, 291 (1984); E. Alvarez-Lacalle, J. Ortín, and J. Casademunt, Phys. Rev. E 74, 025302(R) (2006).

[23] L. M. Martyushev and A. I. Birzina, J. Phys. Condens. Matter 20, 045201 (2008).

[24] E. Pauné, Ph.D. thesis, University of Barcelona, 2002. 